\title{
OWN Survey: a spectroscopic monitoring of Southern Galactic O and WN-type stars
}

\author{
Rodolfo H. Barbá ${ }^{1}$, Roberto Gamen ${ }^{2}$, Julia I. Arias ${ }^{1}$ \\ and Nidia I. Morrell ${ }^{3}$ \\ ${ }^{1}$ Departamento de Física y Astronomía, Universidad de La Serena, Chile \\ email: rbarba@userena.cl \\ ${ }^{2}$ Instituto de Astrofísica de La Plata, CCT La Plata-CONICET, La Plata, Argentina \\ ${ }^{3}$ Las Campanas Observatory, Observatories of the Carnegie Institution of Washington, La \\ Serena, Chile
}

\begin{abstract}
We summarize the status and results of the OWN Survey, a high-resolution monitoring program of Southern Galactic O- and WN-type stars, after twelve years of observing campaign.
\end{abstract}

Keywords. binaries: close, binaries: spectroscopic, stars: early-type, stars: Wolf-Rayet, surveys

\section{Introduction}

The High-resolution spectroscopic monitoring survey of Southern Galactic O- and WNtype Stars (OWN Survey) project has its foundational ideas in the school of stellar spectroscopy that is consolidated in the Observatorio Astronómico de La Plata (Argentina). This school grew specifically in the study of early-type stellar atmospheres, massive stars and binary systems. It is worth mentioning the pioneering work of those years carried out by Dr. Jorge Sahade in the area of interacting binaries, Dr. Virpi Niemelä on massive binaries, and Dr. Hugo Levato in binary systems in open clusters, together with a strong group of students and collaborators.

It should be noted that the OWN Survey was born about 13 years ago, as a result of the stimulating talks with Dr. Virpi Niemela, leader of the "Massive stars Research Group" at La Plata, and with the dedicated work of Dr. Roberto Gamen, at that time, postdoc at the Astronomy Group of the Universidad de La Serena.

The idea of the spectroscopic monitoring survey arises in the lack of full knowledge of the multiplicity status of many optically bright O-type stars in the Milky Way. By the year 2005, it was known that the distribution of binary periods in massive stars differed markedly from that of periods of low- and intermediate-mass stars. Mason et al. (1998, 2009) show a period distribution for O-type stars which is clearly bimodal, in contrasts with the period distribution of stars of solar type stars (Duquennoy \& Mayor, 1991). Since then it was suspected that this bimodality could be a consequence of a strong observational bias produced by the limitations of the techniques used in the detection of binaries in massive stars. Such bimodality posed the existence of a gap in the period distribution, expanding from periods of one month and to 20,000 years. It is illustrative, to bear in mind the ignorance about period distribution of massive binary stars has periods has an enormous impact in modeling of massive star formation and evolution.

With the aim of contributing to the knowledge of the multiplicity status of massive stars is that $O W N$ Survey was born in 2005. Our approach is clearly empirical from the point of view of the determination of radial velocities $(\mathrm{RVs})$ of a selected sample of massive stars during timescales of years. Parallel to the last years, considerable progress 
Table 1. Instrument configurations used in $O W N$ Survey

\begin{tabular}{l|c|c|c|}
\hline Observatory & Telescope & |Spectrograph & Resolution \\
\hline CASLEO & $2.15 \mathrm{~m}$ "Jorge Sahade" & REOSC & 15,000 \\
Las Campanas & $2.5 \mathrm{~m}$ "du Pont" & Echelle & 48,000 \\
La Silla & $2.2 \mathrm{~m}$ & FEROS & 48,000 \\
CTIO & $1.5 \mathrm{~m}$ & BME & 50,000 \\
\hline
\end{tabular}

has been made in the knowledge about the multiplicity status of massive stars using different observational techniques, such as the case of spectroscopy (Chini et al. 2012; Kobulnicky et al. 2012; Sana et al. 2012; Almeida et al. 2017), adaptive optics (Turner et al. 2008; Close et al. 2012), speckle interferometry (Mason et al. 1998, 2009, "lucky imaging" (Maíz Apellániz 2010; Barbá et al. 2017), optical interferometry (Sana et al. 2011, 2013, 2014; Sánchez-Bermúdez et al. 2013, 2014), Fine Guidance Sensor on board the Hubble Space Telescope (Nelan et al. 2014; Aldoretta et al. 2015), among others.

\section{Goals of OWN Survey}

The main goal of the OWN Survey is to set the multiplicity status for bright southern $\mathrm{O}-$ and WN-type stars through spectroscopic and radial velocity monitoring. Also, detection of spectroscopic variability is an additional outcome, for example as the case of the Oe star HD 120678, which underwent a shell-like episode in 2008 documented by the OWN survey (Gamen et al. 2012), the discovery of strong spectral variations in the magnetic Of?p star CPD -28 2561 (Wade et al. 2015), or those variations produced in interacting binaries. Another important goal is to establish a set of massive stars without close companions useful for the testing stellar models for single stars, as is the case of abundance studies in stars without interactions with close companion (e.g. Martins et al. 2016).

The star sample for the $O W N$ Survey has been defined based on the first version of the Galactic O star Catalog" (GOSC v1.0, Maíz Apellániz 2004), and the "VIIth Catalogue of Galactic Wolf-Rayet stars" and annexes (van der Hucht 2001). That sample consists of 180 O-type stars and 58 WN-type stars without a clear evidence of binarity and stars with scarce RV information. At the present, the sample of O-stars under spectroscopic monitoring by the OWN Survey has been expanded to 205 stars, to include some additional targets monitored in the "Galactic O-star Spectroscopic Survey" (GOSSS, Sota et al. 2011, 2014; Maíz Apellániz et al. 2012, 2016) and the "Near-Infrared Photometric Monitoring of Galactic Star Forming Regions" (NIP, Barbá et al. 2011).

Observations are carried out at different facilities in the Chile and Argentina. Table 1 shows the instrumental configuration used and their typical resolutions. After twelve years of monitoring, we have obtained more than 7,000 high-resolution spectra, most of them having signal-to-noise over 200 (Fig. 1. The whole sample of 205 O-stars has been observed at least in five different epochs, having $50 \%$ of the sample 17 spectra or more. Additionally, in the framework of our project, we have observed an important number of known binaries in order to improve their orbital solutions and also to detect apsidal motions. This parameter is a very good proxy to derive absolute masses of the binary components, using stellar structure models (e.g. Ferrero et al. 2013; Ferrero 2016). Thus, the total number of stars monitored is almost 300 .

Some additional O-type stars are observed less frequently, using using intermediateresolution spectrographs. This also holds for the sample of $58 \mathrm{WN}$-type stars.

The $O W N$ Survey is actually a project tightly coordinated with other similar survey projects, and thus sharing spectroscopic databases and results with the GOSSS (Sota 


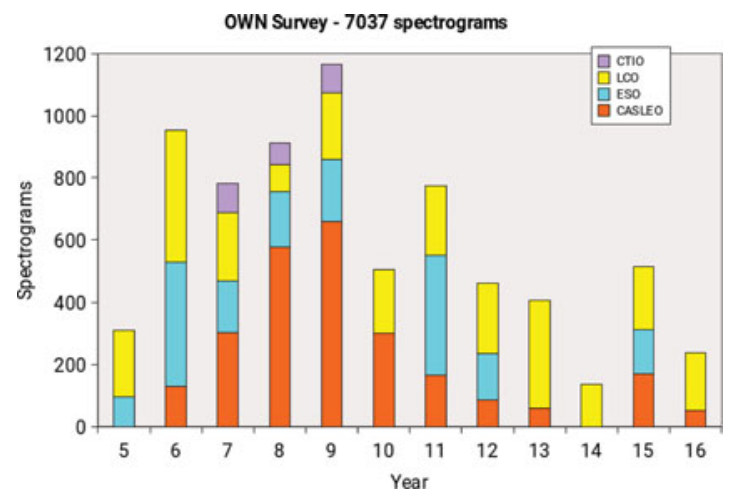

Figure 1. Number of high-resolution spectrograms obtained from different facilities as a function of time.

et al. 2011; Maíz Apellániz et al. 2012), IACOB (Simón-Díaz et al. 2011) and CAFÉBEANS (Negueruela et al. 2015).

\section{Some results of the survey}

From the sample of 205 southern O-stars without a clear spectroscopic signature of binarity, we have determined that 114 stars show RV variations larger than $20 \mathrm{~km} \mathrm{~s}^{-1}$ (typical errors about $1-2 \mathrm{~km} \mathrm{~s}^{-1}$ ), 59 stars show RV variations in the interval $10-20 \mathrm{~km} \mathrm{~s}^{-1}$, while 43 stars show variations below $10 \mathrm{~km} \mathrm{~s}^{-1}$. It is interesting to note that 63 stars display RV variations over $50 \mathrm{~km} \mathrm{~s}^{-1}$, indicative of an important number of binaries in the sample. Binaries can also contribute to the interval of $10-20 \mathrm{~km} \mathrm{~s}^{-1} \mathrm{RV}$ variations. Simón-Díaz (2017) has called the attention to the fact that pulsations in massive stars can potentially be confused with binary motion. Hence, low amplitude RV variations due to binarity should be defined through systematic spectroscopic monitoring, with increased cadence. This issue has a large impact in the completeness of our knowledge about systems with low-mass ratios and/or orbital inclinations.

The main result of the OWN Survey is the detection of 39 SB1, 47 SB2, and 16 SB3 systems, while 60 stars present RV variations larger than $10 \mathrm{~km} \mathrm{~s}^{-1}$, and 43 stars can be classified as "single". We have defined as single those stars with RV variations below $10 \mathrm{~km} \mathrm{~s}^{-1}$, and without a clear periodic signal. From our observations of these 102 spectroscopic binaries, we have determined 85 RV orbits, 55 of which are derived for first time. Figure 2 summarizes the main results of the $O W N$ Survey compared to the RV information available for those 205 O-stars before 2005.

In the case of $58 \mathrm{WN}$-type stars comprised in the survey, most of the results have been presented in the $\mathrm{PhD}$ thesis of A. Collado (2014). We have determined that $22 \mathrm{WN}$-stars present RV variations over $30 \mathrm{~km} / \mathrm{s}$, while in 16 stars, variations are below that limit. We have derived the binary status for 20 systems (18 new orbits), splitted in equal amounts between SB1 and SB2. Taking into account the all known WR binaries, we have counted "only" 68 binary systems with determined periods among a total of 634 WR stars listed in the WR Catalogue compiled by P. Crowther (Rosslowe \& Crowther 2015).

There are mostly two classes of WN-type stars in our sample: those very massive objects, like WR25 or WR21a, and those likely descendant from O-stars, like WR29 or WR36. In both types of WN stars, the detection of binaries is a more complicated task than in O-type stars due to the presence of strong emission lines, specially in the second group of evolved WN-type stars, where also intrinsic variations in the profiles hinder 


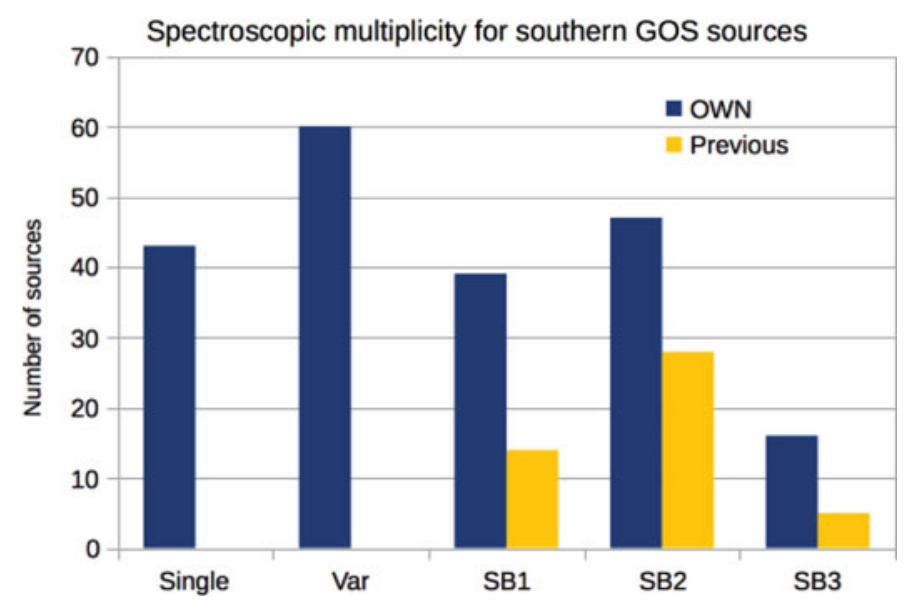

Figure 2. Spectroscopic multiplicity for southern bright GOSC sources. "Previous data" refer to systems known before 2005, starting year of OWN Survey.

the RV variations due to binary motion. Also, we must to take into account that the WR stars in our sample are in general fainter than the O-stars, therefore the spectral resolutions used in our monitoring are a factor of 10 or 20 lower than those used for the monitoring of O-type stars. This is an additional observational issue to take into account when evaluating our knowledge of the binary status of WN-tye stars. Consequently, it is early to conclude any evolutive scenario from the binary frequency in O- and WN-type stars due to the different completeness in our knowledge of both kind of objects. We shall come back to this point in the next section.

\section{About binary star parameter distributions}

We have collected from the literature the available information for all the known Galactic O-type binaries and combined it with our discoveries, bringing the number of known orbits to 220, a good figure for statistical studies. Figure 3 shows the period distribution for all the known Galactic O-type binaries. We have also highlighted the Southern systems and the eclipsing binary systems. The first evident conclusion is that for periods of a few days the number of Southern binaries doubles or even triplicates the number of Northern binaries. This should be compared to the 0.67 value expected for the North to South number ratio of O-type stars as derived from the GOSC (Fig. 3 right). For very long periods (thousands of days), the ratio is higher (0.75) due to observational efforts by astronomers using different techniques as enumerated in the Introduction. Therefore, we can conclude that the Southern Galactic O-type stars are more or less evenly surveyed for multiplicity in order to cover the Mason's gap in binary period distribution. Meanwhile, in the Northern hemisphere the picture is not the same, dedicated high-resolution spectroscopic monitoring surveys of massive stars are not common, and this is the niche that CAFE-BEANS and IACOB surveys are exploring.

The distribution of mass ratios $(q)$ for all SB2 systems (Fig.4) in the sample considered shows a more or less even distribution between 0.3 to 1.0, except for small differences between bins, which may be explained as statistical fluctuations due to the low numbers of known SB2 systems. Comparing with the "Southern" sample, we can note that the distributions are essentially the same, with a very tiny peak for $q \sim 0.5$. For the eclipsing binaries seems to have a maximum in the bin $q=.5$, but it needs to be analyzed if in close binaries this mass-ratio is favored. Also, the sharp drop in the number of binaries 

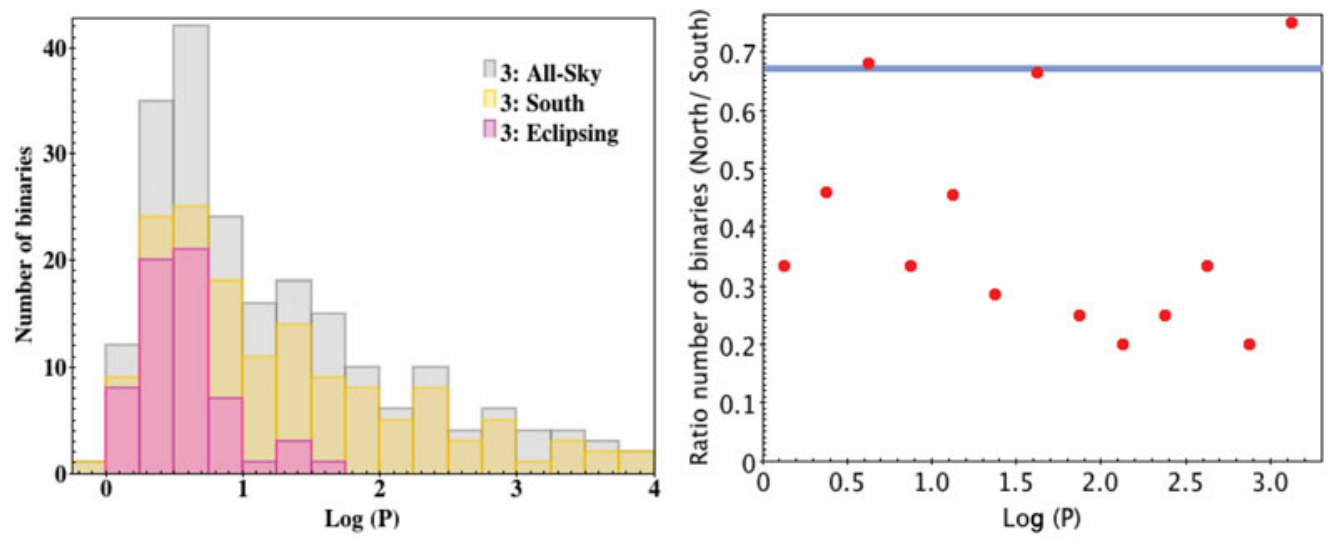

Figure 3. Left: Period distribution for known Galactic binaries. Right: North to South number ratio, the blue line represents the ratio derived for all O-stars in both hemispheres.

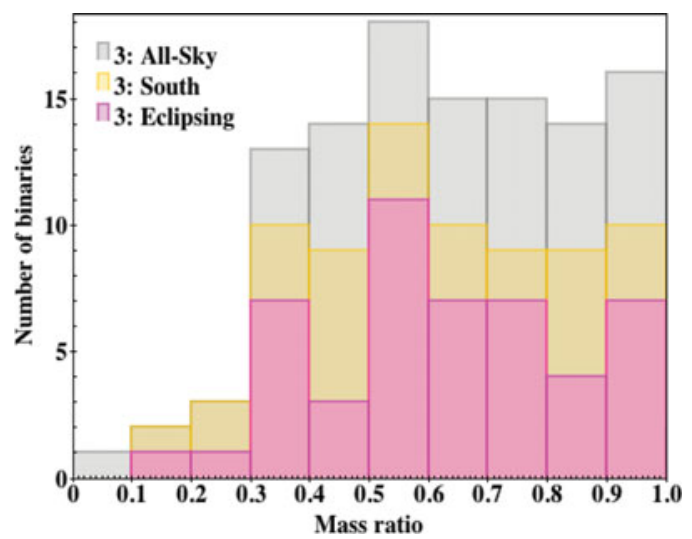

Figure 4. Mass-ratio distribution for all the known Galactic O-type stars.

with $q<0.3$ needs to be explored. It could be related to an observational bias, because smaller mass-ratio also points to smaller luminosity-ratio, and then larger difficulties in spectroscopically detecting the secondary component. There may exist also systems with moderately long periods (weeks or months), where one of the component is a very fast rotator. In those cases, the line profiles of the fast rotator are immersed inside the continuum of the narrow-lined component, and then very hard to separate. These issue needs to be explored through the monitoring of some short period SB1 systems with highest achievable resolution and signal-to-noise.

The period-eccentricity distribution for all O-type binary systems is presented in Figure 5. The lack of systems with very high eccentricities for periods of a few days is expected because the limit imposed for the size of stars are of the same order of the orbital separations at periastron passage. Taking into account some specific spectral types, we can note that late-type supergiants show an enhancement for periods between 3 and 50 days and eccentricities larger than 0.3 , compared with main-sequence binaries. This fact perhaps is indicative of evolutive signatures in binaries, for example, SB1 systems with compact companions, as in the case of HD 74194, a high-mass SB1 fast X-ray transient with a $P=9.54$ days and $e=0.63$ (Gamen et al. 2015). Moreover, it is interesting to note the relatively low number of binaries with periods longer than 100 days and eccentricities lower than 0.2 . This effect in part could be explained by the difficulties in detecting 

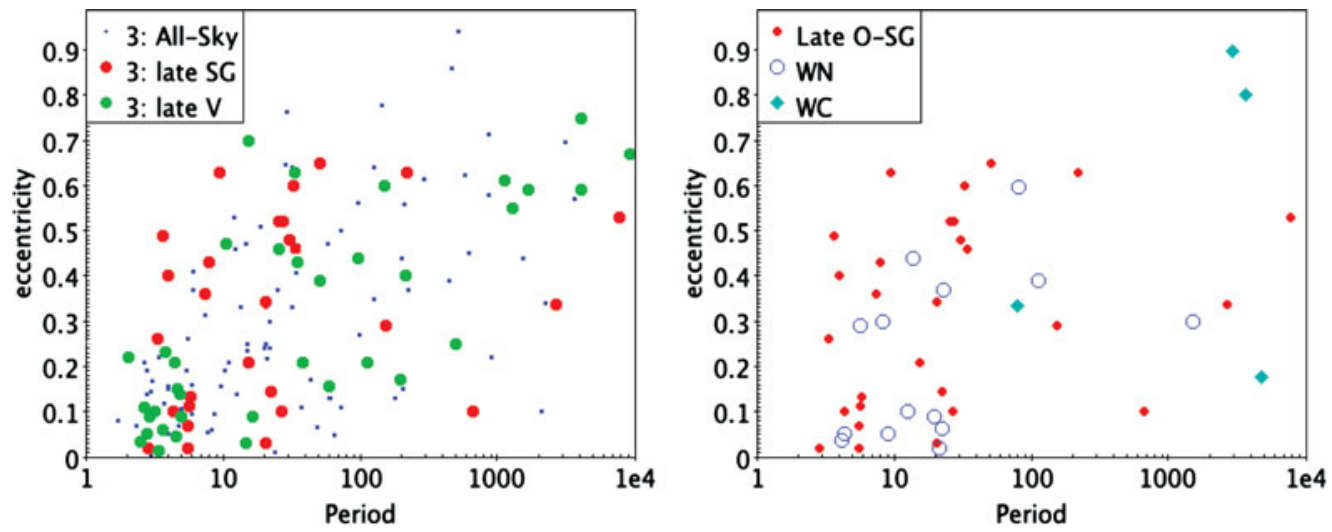

Figure 5. Eccentricity-period distribution for all Galactic O-type binaries (right), and including the WR stars (left).

low amplitude binaries with long periods, but also could be related to the mechanism involved in the formation of this kind of binaries.

The comparison with the $P-e$ distribution between late O-type supergiants and $\mathrm{WN}$ and WC-type stars (Fig. 5 shows that WN stars follow a similar distribution to that of O-type supergiants. Although the number of WC-type stars with known eccentricities is low, it is noticeable the lack of systems with periods below 80 days.

\section{The multiplicity status of O-type stars}

The multiplicity status of O-type stars should be studied considering the differences in spectral types and luminosity classes if we wish to understand how to the systems are formed and how they evolve. Sana et al. (2012) concluded that the binary interaction dominates the evolution of massive stars and their calculations propose that $24 \%$ of all the systems do merge. Therefore, beyond the observational biases, we must to take into account that binary interaction could affect the true number of binaries in each spectral type and luminosity class. We have started the study of the distribution of binaries taking into account both parameters. A zero order approach for the analysis can be done counting the number of spectroscopic binaries per luminosity class. Table 2 displays the relative frequency of spectroscopic binaries for 411 stars (205 from the $O W N$ Survey) for four different luminosity classes, i.e. supergiants, bright giants, giants, and main-sequence plus subgiants. Obviously, this is a coarse approach to describe the evolutionary status of these stars. For example, an O2 If* star (i.e. HD 93129 A) is more related to main-sequence stars than an O8.5 Ib-II(f)p star (i.e. HD 74194). Taking into account this caveat, we can note that the relative numbers of "single" stars show maxima for luminosity classes I, and II, reinforcing the idea that some supergiants could be merger products from the evolution of close binaries, or others became single stars after the disruption of a binary due to the evolution of the former primary and ulterior explosion as supernova, or the dynamical ejection from a massive cluster. Another interesting feature is the high number of SB2 systems for the luminosity class III. This definition of this luminosity class is based on the appearance the HeII 4686 absorption line. We can speculate that the origin of this observational feature, can be related to the presence of interacting binaries, where line profiles may be distorted. There is also the possibility that some of these "SB2 systems" are not real binaries, but fast rotators with profile variations induced by pulsations. 
Table 2. Frequency of the spectroscopic multiplicity status for 411 Galactic O-type stars in function of luminosity.

\begin{tabular}{l|l|l|l|l|}
\hline Status & $\mathbf{I} / \mathbf{a} / \mathbf{a b} / \mathbf{b}$ & II & III & $\mathbf{I V}-\mathbf{V}-\mathbf{V z}$ \\
\hline Single & 0.55 & 0.63 & 0.31 & 0.40 \\
SB1 & 0.23 & 0.17 & 0.18 & 0.20 \\
SB2 & 0.17 & 0.11 & 0.37 & 0.29 \\
SB3 & 0.05 & 0.09 & 0.14 & 0.11 \\
\hline
\end{tabular}

Table 3. Spectroscopic multiplicity status for late O-type and early B-type supergiants

\begin{tabular}{|l|c|c|c|}
\hline SpT & No. of Stars & No. of SB & Frequency \\
\hline O7 & 20 & 11 & 0.57 \\
O8 & 19 & 9 & 0.52 \\
O9 & 63 & 24 & 0.38 \\
B0 & 260 & 11 & 0.04 \\
\hline
\end{tabular}

We can consider that the main-sequence stars in the spectral range between O5O9.7 have masses in the range between 30-40 to 13-16 $\mathrm{M}_{\odot}$ (c.f. Martins et al. 2005). Typical evolutionary scenarios (e.g. Brott et al. 2011, Ekström et al. 2012) for single stars in this mass range predict that the O-stars will evolve as late-O or early B-type supergiants in a timescale of few mega-years (although hypergiants may have different origin). It would thus be of great interest to compare the multiplicity status of these supergiants. Such status is relatively well known for late O-type supergiants but this is not the case for early B-type supergiants. We have compiled the information about the multiplicity status of early B-type supergiants surveying in detail the literature for more than 1,500 stars extracted from the Skiff's recompilation of spectroscopic classification of stars (Skiff 2014). The results are surprising, only 63 stars are classified as spectroscopic binaries. Table 3 shows the spectroscopic binary frequencies derived from our studies and the literature for late-O and B0 supergiants and bright giants. The sharp drop in the number of binaries between spectral types O9.7 and B0 is noticeable. It may be produced by an observational bias due to the lack of dedicated spectroscopic monitoring programs for B0 supergiants, but also we must to explore the possibility of evolutive scenarios in action like binary mergers, binary disruptions, etc.

\section{Perspectives on multiplicity of massive stars}

When the $O W N$ Survey program started, one of our expectations was detecting systems with periods of tens of days or even longer, but one of our surprises was the discovery of bright close binaries in hierarchical triple systems as the cases of HD $92206 \mathrm{C}$ (Campillay et al. 2007) or Herschel 36 (Arias et al. 2010).

Sana et al. (2012) have suggested that about $70 \%$ of all massive binaries interact with their companions, leading to mergers in one-third of cases. Therefore, the high number of spectroscopic hierarchical triple systems composed by a close binary and a massive companion in a wider orbit opens new evolutive scenarios, which have not been explored yet. For example, in an important number of cases we should to consider a double interacting scenario, where the close binary system evolves to a merger and then interacts with the third companion. Obviously, the different scenarios will depend on the different system parameters, such as mass-ratio of the close binary, mass of the tertiary companion, separation between different components, orbit eccentricities, spins, etc.

The $O W N$ Survey has brought new interesting observational facts about the complexity of the massive binary stars. 


\section{Acknowledgments}

RHB thanks support from project FONDECYT Regular No 1140076.

\section{References}

Aldoretta, E. J. et al. 2015, AJ, 149, 26.

Almeida, L. A. et al. 2017, A\&GA, 598, A84.

Arias, J. I. et al. 2010, ApJ, 710, L30.

Barbá, R. H. et al. 2011, Bol. Asoc. Arg. Astron., 54, 85.

Barbá, R. H. et al. 2017, in preparation.

Brott, I. et al. 2011, A\&SA, 530, A115.

Campillay, A. et al. 2007, VI Reunion Anual Sociedad Chilena de Astronomia (SOCHIAS), held in Valparaiso, Chile, 7-9 Nov. 2016, 63.

Chini, R., Hoffmeister, V. H., Nasseri, A., Stahl, O., \& Zinnecker, H. 2012, MNRAS, 424, 1925.

Close, L. M. et al. 2012, ApJ, 749, 180.

Collado, A. 2014, PhD. Thesis (Universidad Nacional de San Juan, Argentina).

Duquennoy, A. \& Mayor, M. 1991, A\&̈A, 248, 485.

Ekström, S. et al. 2012, A $E A$ A, 537, A146.

Ferrero, G., Gamen, R., Benvenuto, O., \& Fernández-Lajús, E. 2013, MNRAS, 433, 1300.

Ferrero, G. 2016, PhD. Thesis (Universidad Nacional de La Plata, Argentina).

Gamen, R. et al. 2012, AछA, 546, A92.

Gamen, R. et al. 2015, A\&BA, 583, L4.

Kobulnicky, H. A. et al. 2012, ApJ, 756, 50.

Maíz Apellániz, J., Walborn, N. R., Galué, H. A., \& Wei, L. H. 2004, ApJS, 151, 103.

Maíz Apellániz, J. 2010, Aछ̛A, 518, A1.

Maíz Apellániz, J. 2012, $A S P C, 465,484$.

Maíz Apellániz, J. et al. 2016, ApJS, 224, 4.

Martins, F., Schaerer, D., \& Hillier, D. J. 2005, A\&\&A, 436, 1049.

Martins, F., Simón-Díaz, S., Barbá, R. H., Gamen, R. C., \& Ekström, S. 2016, (A\&A), in press (2016arXiv161105223M).

Mason, B. D., Gies, D. R., Hartkopf, W. I., Bagnuolo, W. G. Jr., ten Brummelaar, Th., \& McAlister, H. A. 1998, AJ, 115, 821.

Mason, B. D., Hartkopf, W. I., Gies, D. R., Henry, T. J., \& Helsel, J. W. 2009, AJ, 137, 3358.

Negueruela, I. et al. 2015, Highlights of the Spanish Astrophysics, 8, 524.

Nelan, E. P., Walborn, N. R., Wallace, D. J., Moffat, A. F. J., Makidon, R. B., Gies, D. R., \& Panagia, N. 2014, AJ, 128, 323.

Rosslowe, C. K. \& Crowther, P. A. 2015, MNRAS, 447, 2322.

Sana, H. et al. 2011, ApJ, 740, L43.

Sana, H. et al. 2012, Sci, 337, 444.

Sana, H. et al. 2013, A\&A, 553, A131.

Sana, H. et al. 2014, ApJS, 215, 15.

Sánchez-Bermúdez, J., Schödel, R., Alberdi, A., Barbá, R. H., Hummel, C. A., Maíz Apellániz, J., \& Pott, J.-U. 2013, A\&A, 554, L4.

Sánchez-Bermúdez, J., Alberdi, Schödel, R., Hummel, C. A., Arias, J. I., Barbá, R. H., Maíz Apellániz, J., \& Pott, J.-U. 2014, A\&SA, 567, A21.

Simón-Díaz, S., Castro, N., García, M., Herrero, A., \& Markova, N. 2011, Bull. Soc. Roy. Sci. Liege, 80, 514.

Simón-Díaz, S. 2017, IAU Symposium, 329, this volume.

Skiff, B. A. 2014, Vizier Online Data Catalogue: Catalogue of Stellar Spectral Classification.

Sota, A. et al. 2011, ApJS, 193, 24.

Sota, A., Maíz Apellániz, J., Morrell, N. I., Barbá, R. H., Walborn, N. R., Gamen, R. C., Arias, J. I., \& Alfaro, E. J. 2014, ApJS, 211, 10.

Turner, N. H., ten Brummelaar, Th.A., Roberts, L. C., Mason, B. D., Hartkopf, W. I., \& Gies, D. R. $2008, A J, 136,554$.

van der Hucht, K. A. 2001, New Astron., 45, 135.

Wade, G. A. et al. 2015, MNRAS, 450, 2822. 